\title{
Observation-Oriented Causal Discovery for Cultivation Abandonment of Rice Terraces: Focusing on an Effect of Cultural Endemism on Decision-Making in Toraja, Indonesia
}

\author{
Ayako Oide1,2*, Osamu Kozan1, Tomoko Doko3,4, Wenbo Chen ${ }^{3,4}$ \\ ${ }^{1}$ Center for Southeast Asian Studies, Kyoto University, Kyoto, Japan \\ ${ }^{2}$ Graduate School of Asian and African Area Studies, Kyoto University, Kyoto, Japan \\ ${ }^{3}$ Nature \& Science Consulting Co. Ltd., Yokohama, Japan \\ ${ }^{4}$ Keio Research Institute at SFC, Keio University, Fujisawa, Japan \\ Email: *oideayako@gmail.com
}

Received 18 January 2016; accepted 21 February 2016; published 24 February 2016

Copyright (C) 2016 by authors and Scientific Research Publishing Inc.

This work is licensed under the Creative Commons Attribution International License (CC BY). http://creativecommons.org/licenses/by/4.0/

(c) (i) Open Access

\section{Abstract}

Previous discussion about the factors of the expanding trend of abandoned cultivation had focused only on universal factors and lacked evaluation of the regionality of the phenomenon. This paper demonstrated the Toraja's regional characteristics and the influence of cultural endemism on decision-making about abandoning cultivation by an observation-oriented approach. Based on a causal framework constructed by field observation and geo-spatial data generation, an adjustment for overt covariates using the multivariate logistic regression model to draw the causal effect from hidden covariates was examined in two rice terraces with different water systems, i.e. irrigated field and rain-fed field. The result of sub-group analysis revealed that decisions about abandoning cultivation in Toraja were greatly associated with disadvantageous factors for intensive farming, i.e. "number of adjacent fields" and "soil erosion" rather than advantageous factors, i.e. "area of field" and "distance to roads". Moreover, the result of interaction analysis which controlled the effect of topography revealed the powerful effect of particular decision factors only in rain-fed rice terrace: the "distance to roads" factor's fairly negative contribution on abandoning cultivation (Odds ratio $=9.94 \mathrm{E}-01$, $P$ value $=2.03 \mathrm{E}-11$ ), as well as the "number of adjacent field" factor's positive contribution on abandoning cultivation (Odds ratio $=1.13 \mathrm{E}+00, \mathrm{P}$ value $=$ 3.65E-04). Given the evidence from the explanation of these results by customary laws and land inheritance system for each site, therefore, it could be concluded that the screening and detection of cultural endemism's influence was achieved using the algorithm this paper proposes.

\footnotetext{
${ }^{*}$ Corresponding author.
}

How to cite this paper: Oide, A., Kozan, O., Doko, T. and Chen, W.B. (2016) Observation-Oriented Causal Discovery for Cultivation Abandonment of Rice Terraces: Focusing on an Effect of Cultural Endemism on Decision-Making in Toraja, Indonesia. Agricultural Sciences, 7, 100-113. http://dx.doi.org/10.4236/as.2016.72010 


\section{Keywords}

\section{Cultivation Abandonment, Decision-Making, Rice Terrace, Observation-Oriented, Multivariate Logistic Regression, Sub-Group, Interaction, InSAR, Soil Erosion, GIS}

\section{Introduction}

\subsection{Background}

Cultivation in mountainous regions has a high potential for abandonment because of such areas' unfavorable topography for intense labor and the marginality from the socio-economic activities [1]-[4]. In Toraja, mountainous region of Sulawesi, Indonesia, the rice terrace is a distinctive landscape related to ritual and culture [5]. Oide et al. (2015) clarified the centrality of rice cultivation in the Toraja people's livelihood [6]. The rice cultivation of Toraja is seen as a communal collective action because such areas have linked irrigation systems and water basin clusters due to their topography. However, as with other mountainous region, a trend toward abandoning cultivation of rice terraces is notably prominent in Toraja region currently. Studies of behavioral science have pointed out that the motivation to engage in collective acts is influenced by the perceived value placed on the outcome [7] [8]. Although most studies evaluated the rice cultivation in Asian monsoon region by the various ecosystem functions [9], rice cultivation in Toraja is highly valued due to a strong linkage to culture and rituals. In addition, as Molden et al. (2000) write, the perception of the contribution of rice cultivation practices and paddy irrigation often differs among people because society places widely different values on uses of water and land [10]. Thus there is a need to clarify regional differences in local people's decision-making about rice cultivation and effect of regional culture and ritual on them.

However, the causal factors of the expanding trend of abandoned cultivation are commonly discussed from a global overview, focusing on the trends to socio-economics and environment. Numerous studies have pointed out the universal risk factors: topography [11], soil related indices [12] [13], climate [14] and accessibility [15]. Hatna \& Bakker (2011) as in [16] have quantitatively evaluated these risk factors for cultivation abandonment on a broad scale using geo-spatial information. Contrary to these findings from prior studies, the actual situation of cultivation abandonment differs in regional scale, and it cannot be explained equally with the factors mentioned above. Therefore, in order to generate the practical policy recommendations on a prevention of degradation of mountainous area, it is necessary to discover the causal factors contributing to the abandonment of cultivation in regional scale by on-site observation.

An observational study is an empirical but nonexperimental investigation of the effects caused by a treatment [17]. In order to estimate the causal effects of subjects' outcomes in observational studies, biases are needed to be controlled. However because no established theory about regional decision-making for abandoning cultivation exists, a structure of causal association has to be first constructed by field observation prior to the analysis.

\subsection{Research Objectives}

There are two objectives in this study. The first is to evaluate the influence of cultural endemism on local people's decision-making about abandoning cultivation and to consider the regionality of Toraja society. The second is to demonstrate an observation-oriented approach to describe the regional characteristics of decisionmaking about cultivation abandonment by integrating qualitative field observations with statistical quantification via geo-spatial information and technologies. The specific research questions to be answered are as follows:

1) What kind of regional characteristics of decision-making about abandoning cultivation exist and how do they differ between regions?

2) What kind of influence does cultural endemism exert on decision-making about abandoning cultivation and how does it differ between regions?

To approach the above-mentioned research questions, the following conditions based on field observations were assumed in order to build an examination algorithm for overall analysis:

1) Local people's decision-making about abandoning cultivation can be explained by particular decision factors.

2) Local people’s decision-making about abandoning cultivation can potentially be influenced by topographic 
conditions and cultural endemism.

On this basis, this paper developed a quantitative evaluation algorithm to discover the causal association of cultural endemism with cultivation abandonment of rice terraces by integrating the qualitative field observations and quantitative statistical evaluation. In the end, the results of analysis on Toraja's cultural endemism and the application of an integrative approach were discussed.

\section{Method}

\subsection{Causal Framework Construction by On-Site Field Observations}

As R. A. Fisher advised Cochran to "make your theories elaborate" in response to the question of what can be done in observational studies to clarify the step from association to causation [18], the initial process for evaluating causal effect in observation study is elaboration of theory. Although prior research about Toraja concluded the association of cultural and rituals with rice cultivation from a qualitative perspective [5], little study has been done to actually explore the causal association of them using scientific or statistical methods. In a situation where no theory established about the regional causation of cultivation abandonment, what need to be done first is to construct the causal structure based on a primary on-site survey prior to statistical causal discoveries.

Figure 1 illustrates the causal framework in this study. In this paper, regional rituals and culture associated with rice cultivation are defined as "cultural endemism". Based on a field observations and interviews, it was assumed that local people's decisions for abandoning cultivation were represented by particular factors defined as "decision factors". The detected decision factors were summarized as four terms: 1) area, 2) distance to road, 3) number of adjacent rice terraces, and 4) soil erosion. These decision factors were further summarized into two groups in terms of interest in intensive farming: advantageous or disadvantageous factors. Because these factors were all land-related indices, in addition to the fact that the site selection process lacked spatial random sampling, like other observational research, the results of analysis cannot be summarized with a simple comparison as with experimental research.

Consequently, the decision factors are presumed to be potentially influenced not only by cultural endemism, but also by topographic conditions. Thus topography and cultural endemism were then addressed as potential confounders for both decision factors and outcome. In order to detect the hidden causal effect of cultural endemism which has not been measured, the effect of topography needs to be removed. The adjustment for topographic indices was achieved by the geo-spatial data generation using GIS and remote sensing techniques.

\subsection{Integrative Approach via Geo-Spatial Information and Technologies}

The present study integrates several methods by the application of the geo-spatial information and techniques. The approach consists of major three parts: field observations and interviews (2.2.1), geo-spatial data generation (2.2.2), and the examination of the logistic regression model (2.2.3). The results of logistic regression were discussed with factors of sub-regional cultural endemism that were revealed by field observations.



Figure 1. Causal structure detected by field observation. 


\subsubsection{Field Observations about Cultural Endemism}

To clarify the sub-regional cultural endemism of two study sites, a field interview survey about the differences in rice cultivation and its related culture between two study sites was conducted through a semi-structured interview method [19] from September to November 2014. The respondents were heads of households or senior family members who were landowners at the observation sites, and were aged 50 or more. The survey was conducted through conversations with a total of 40 respondents, 20 from a village located near site $\mathrm{A}$ and another 20 from a village near site B. Individual interviews lasted approximately two hours. The interview items about the variety of rice, the consumption and usage of the rice, the harvesting times during the year, the planting and harvesting methods, land tenure, and inheritance systems were categorized as "cultural endemism".

\subsubsection{Geo-Spatial Data Generation}

To examine logistic regression, the local people's detected decision factors were quantified by a Geographic Information System (GIS). The indices for soil erosion and topography were generated by a radar technique of remote sensing.

Interferometric Synthetic Aperture Radar (InSAR) is a radar technique that generates a map of topography and ground displacement by separating the related phase terms [20]. The topography-related phase term was estimated by an independent pair of Synthetic Aparture Radar (SAR) images acquired within a short observation interval from a slightly different orbit configuration, which were combined to exploit the phase difference of the signals. Since the interferometric phase is sensitive to both surface topography and the coherent movement of the scatter in the line of site direction between the acquisitions, it is effective in deriving topography or detecting ground surface displacement to a precise millimetric scale. Previous studies have demonstrated InSAR techniques for measuring land surface deformation [21]-[24]. In this study, InSAR techniques were applied to measure the gradual ground displacement caused by soil erosion in rice terraces.

\subsubsection{Logistic Regression to Explain Causality between Factors and Outcomes}

Logistic regression is a statistical model developed by David Cox in 1958 [25]. The resulting odds ratio (OR) can explain the effect of a given variable on the outcome by comparing its magnitude from the threshold of 1 . That is, if the OR equals 1 , the factor does not affect the outcome. If the OR is smaller than 1 , the factor contributes to the outcome negatively, whereas if the OR is higher than 1 , the factor contributes to the outcome positively. The effect of decision factors on abandoning cultivation within and between rice terraces was explained by analysis using a logistic regression model.

However the data used for analysis were the land-related indices, which were based on field observations that lacked spatial random sampling. Thus, it is assumed that the factors are simultaneously influenced by topographic conditions. Therefore, the effect of topography on a particular decision factor has to be removed to estimate the OR of each decision factor precisely, by assuming topographic indices as a covariate. A confounding variable (covariate/confounder) is defined as any variables that is causally associated with the outcome of interest and is non-causally or causally associated with the exposure, but is not an intermediate variable in the causal pathway between exposure and outcome [26].

In this paper, a two-stage evaluation process controlled the effects of topography. First, in order to evaluate the regional characteristics of decision factors, the non-adjusted OR was estimated through sub-group analysis using a univariate logistic regression model. Second, the adjusted OR of each decision factor was estimated by interaction analysis using logistic regression, which is used to control the covariate effect of topographic indices, which were detected by the Mann-Whitney $U$ test for the differences in topographic conditions for each decision factor.

\subsection{Study Site}

The North Toraja region (Latitude: $3^{\circ} \mathrm{S}$, Longitude: $120^{\circ} \mathrm{E}$ ) is a marginal area of South Sulawesi and is located in a mountainous region. Its elevation is 800 meters to 2000 meters. Its total area is 115,147 hectare, and its population is 222,400. The region consists of many small water basins, which are traditionally cultivated on terraced paddy fields and have a traditional water distribution system, which draws water from mountain streams in the upper fields and drains into the lower fields. Based on field interviews and observations, it was revealed that the culture related to rice cultivation in Toraja varied from one sub-regional basin to another. Moreover, these differences in regional culture are especially prominent between sites with different water systems. In order to ex- 
plain the effect of cultural endemism quantitatively, we must refer to the differences between regions in local people's decision-making about rice terrace cultivation. In this study, therefore, two rice terraces with different water systems are examined. In terms of the specific site locations (see Figure 2).

Site A shows irrigated rice terraces, which consist of 719 rice fields inside 51.62 ha. Site B shows rain-fed rice terraces consisting of 3290 rice fields inside 39.15 ha. Because, each of the rice terraces consists of one culture zone, it enabled a representative explanation about the sub-regional characteristics of each site. In addition, these sites were located on the matched socio-economic condition, that is, both of the sites are located about an hour-motorbike ride from Rantepao city. Therefore it enabled to assume the appeared hidden bias as simply cultural endemism excluding the bias from socio-economics.

\subsection{Data Used}

\subsubsection{Decision Factors Detected by Interview Survey}

The detected decision factors could be explained singly by observing the specific circumstances of rice cultivation of the Toraja regions. The yield from rice terraces depends on the area of rice field. In addition, because of the area's precipitous topographic conditions, the labor intensity considerably depends on the distance to accessible roads. Therefore, the terms "area of field" and "distance to roads" were defined as the "advantageous factors" for intensive farming. On the other hands, because Toraja's customary law places the responsibility for footpaths' slope failure and soil erosion on the upper landowners, "number of adjacent fields" and "soil erosion" were considered negative contributions for intensive farming. Thus these were defined as "disadvantageous factors”.

\subsubsection{Variables for Logistic Regression Model}

The data used to execute logistic regression are described in detail in this section. The process used to generate geo-spatial indices for logistic regression is also described precisely.

(1) Presence or absence of cultivation abandonment as an outcome for estimation

To construct a logistic regression model estimating the abandonment of cultivation as its outcome, the cultivation situation detected by field observation as converted to binarized data by assigning 1 to rice fields with a presence of cultivation abandonment and 0 to those with an absence.

A Pleiades image with a resolution of 2 meters was employed as the background image to eye-sight map the rice terraces. The created rice terrace map was converted into the projected polygon features. This mapping analysis was conducted through the software ArcGIS 10.1.

Field investigation and a GPS survey were conducted from August to October 2014, a period of paddy harvesting at the two study sites, to detect abandoned fields. The criterion for on-site judgement of rice fields was the clear shape of footpaths in the rice fields. Steep slopes that were not terraced were considered slope failures

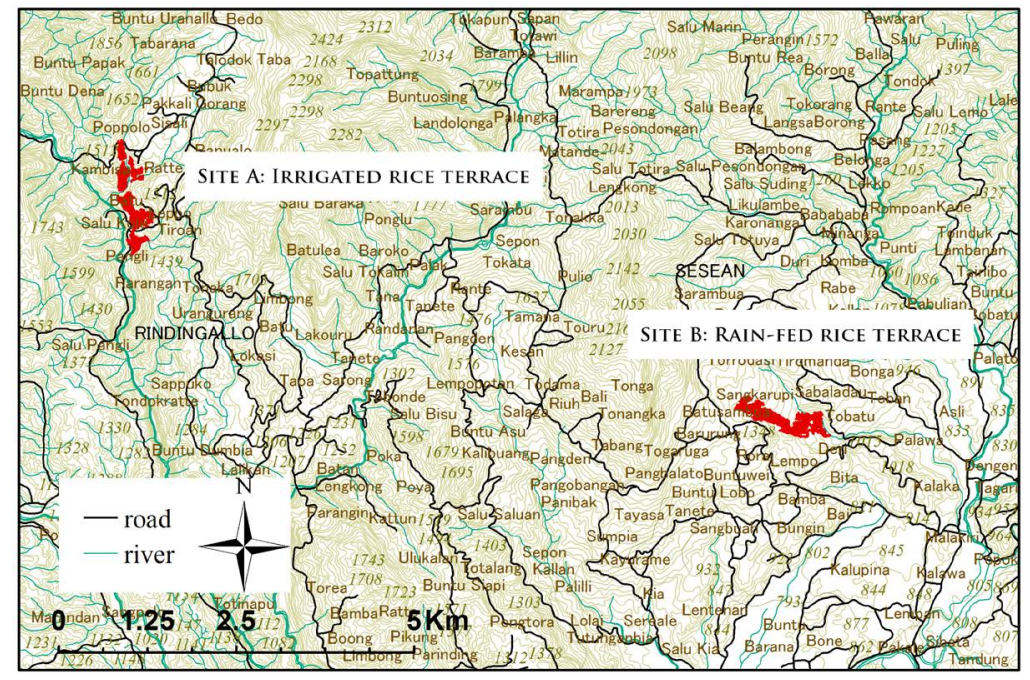

Figure 2. Topography map of observation sites. 
and were excluded from judgment. If the areas had not been cultivated at least during the last cropping term of January 2014 to August 2014, they were considered abandoned. The data about the presence or absence of abandoned cultivation were related to the polygon features of each rice field in ArcGIS 10.1.

\section{(2) Explanatory variables for logistic regression}

The area of each paddy field was calculated by projected polygon shapefile using the Geometry Calculation tool of ArcGIS 10.1. Area was measured in $\mathrm{m}^{2}$. The road data was generated by the GPS field survey. Based on the data, the Euclidean distance from the center point of each paddy field to the road was calculated using ArcGIS 10.1. The number of adjacent rice fields was calculated by counting the overlapping objects using a polygon neighbor analysis tool from ArcGIS 10.1, determined by generating a footpath object with a 1-meter buffer from the boundary of each paddy field according to the field investigations. Soil erosion was represented by the land displacement map calculated by an Interferometric Synthetic Aperture Rader (InSAR) analysis. ALOS PALSAR (Phase Array type L-band Synthetic Aperture Rader) images were used to derive the displacement map and the high resolution Digital Elevation Model (DEM). As shown in Table 1, six pairs of raw SAR single-look complex images that were acquired between May 2008 and February 2011 were registered. These images were in both HH polarization for FBS (Fine Beam Single) mode and HH + HV polarization for FBD (Fine Beam Double) mode. From these pairs, the last pair with a time interval of 45.999 days acquired in December 2010 and February 2011 exhibited the finest coherence with a normal baseline of -337.741 meters and ambiguity height of about 190.026 meters. Therefore the map of displacement and topography resulting from this pair was selected for the soil erosion index. The generated surface displacement image with a resolution of 12 meters has a range from -1 to 1 . Here a negative value indicates the soil loss. Thus, to suit the purpose of indicating the state of soil erosion, the vertical displacement image was converted to a reversal value, and it was extracted for each rice terrace polygon feature by a mean value using the zonal statistic function. The topographic indices of elevation and slope were calculated from the DEM data generated by the InSAR analysis and with a spatial resolution of 12 meters by using the software of ENVI 5.2. The slope was measured in degrees.

\subsection{Statistical Test for Difference in Variables between Two Rice Terraces}

The differences between the two rice terraces were tested by a Mann-Whitney $\mathrm{U}$ test. All $\mathrm{P}$ values are twotailed; those under 0.05 are considered statistically significant. This test was applied to examine if there was a significant difference $(\mathrm{P}<0.05)$ between the two rice terraces regarding all variables of the decision factors' elevation and slope.

\subsection{Evaluation of Decision Factors by Controlling Topographic Confounders in Logistic Regression Model}

In order to remove the effect of topography, two-stage analysis was conducted. First, the non-adjusted OR was estimated by dividing the topographic sub-groups by a mean value of both elevation and slope for each rice terrace (1323.12 m of elevation and 9.94 degree of slope for site $A$, and $1164.07 \mathrm{~m}$ of elevation and 9.92 degree of slope for site B). The results of sub-group analysis indicate the differences in decision factors' effects within regions by topographic conditions. Second, to compare the difference in decision factors' behavior between regions, each OR had to be adjusted by confounding variables in interaction analysis.

Table 1. Registered result of interferogram generation.

\begin{tabular}{cccccccc}
\hline Pair & Master & Slave & Normal baseline (m) & Critical baseline & $\begin{array}{c}\text { Ambiguity } \\
\text { height (m) }\end{array}$ & $\begin{array}{c}\text { Ambiguity } \\
\text { displacement (m) }\end{array}$ & $\begin{array}{c}\text { Time } \\
\text { baseline (Days) }\end{array}$ \\
\hline 1 & May 2008 & Jun 2008 & -506.198 & 11497.757 & 126.668 & 0.118 & 46 \\
2 & May 2008 & Dec 2010 & 280.981 & 11497.757 & 228.196 & 0.118 & 966 \\
3 & May 2008 & Feb 2011 & -80.052 & 11497.757 & 800.967 & 0.118 & 1012 \\
4 & Jun 2008 & Dec 2010 & 712.957 & -15327.686 & 89.961 & 0.118 & 920 \\
5 & Jun 2008 & Feb 2011 & 528.839 & 15327.686 & 121.281 & 0.118 & 966 \\
6 & Dec 2010 & Feb 2011 & -337.741 & 15335.639 & 190.026 & 0.118 & 45.999 \\
\hline
\end{tabular}


Prior to the interaction analysis, the Mann-Whitney U test was examined for the difference in baseline topographic conditions to select the appropriate topographic confounders for each decision factor. The topographic indices with statistical evidence $(\mathrm{P}<0.05)$ were then selected as the confounders and were applied for interaction analysis. The adjusted OR was compared between rice terraces and revealed the influence of cultural endemism on decisions about cultivation abandonment. All analyses were conducted using $\mathrm{R}$ version 3.1.3. The resulting non-adjusted and adjusted OR were plotted to forest plots, which presented the magnitude of effect of each decision factor.

\section{Result}

\subsection{Cultural Endemism of Rice Cultivation}

The results of interviews about cultural endemism of rice cultivation were summarized in Table 2. These cultural endemism factors were considered for discussion with the results of the logistic regression model.

\subsection{Statistical Test for Differences in Variables between Two Rice Terraces}

As Figure 3 shows, the Mann-Whitney U test revealed a significant difference $(\mathrm{P}<0.05)$ between the two rice terraces in all of the risk factors: area, distance to roads, number of adjacent fields, soil erosion, elevation and elevation. Comparing the histogram of both sites shows that a distinctively significant difference in basic cultivation conditions exists in most cases.

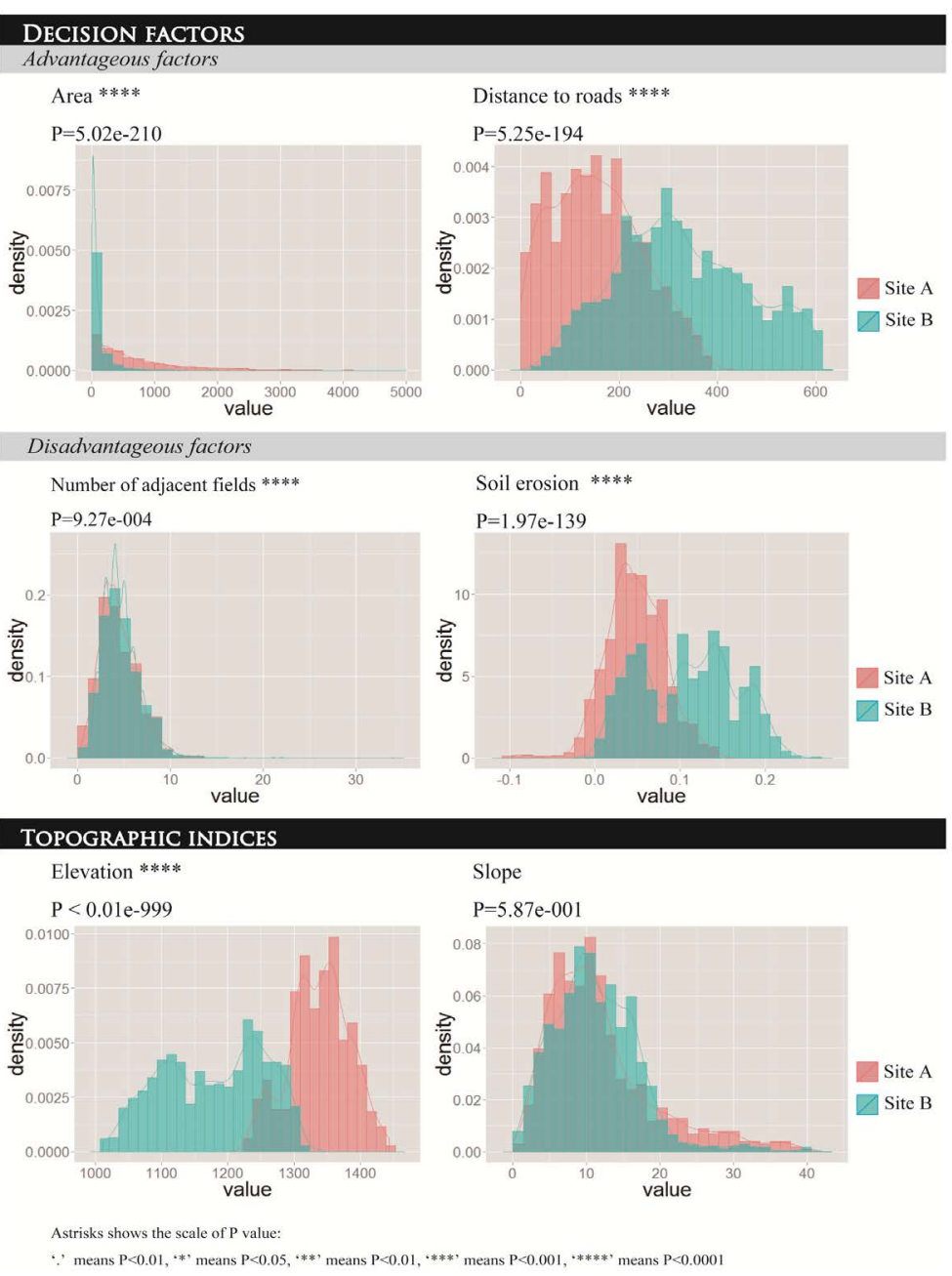

Figure 3. Difference in variables between the two rice terraces. 
Table 2. The differences in cultivation situation and related culture between two sites.

\begin{tabular}{cccc}
\hline & Items & Site A & Site B \\
\hline 1 & Water access & Irrigated & Rain-fed or spring-fed (partially) \\
2 & Produced crop & Hybrid rice & Indigenous rice \\
3 & Consumption of rice & For daily consumption only & For daily consumption, ritual, commercial \\
4 & Harvesting time per year & Double cropping & Single cropping \\
5 & Planting method & Manual transplanting by straight-raw method & Manual transplanting by random method \\
6 & Harvesting implements & Sickle & Ani-ani: small knife \\
7 & Inheritance of land tenure & Single inheritance system & Divided succession system \\
\hline
\end{tabular}

For example, the distribution of area particularly differed between the two sites: the median value of site A was $457.67 \mathrm{~m}^{2}$, whereas that of site B was $56.40 \mathrm{~m}^{2}$. Only a topographic index of slope did not produce any significant difference, revealing similar slope conditions. This indicates the topographic conditions of a rice terrace's location can be described as a similar basin area among various elevations. On the other hand, the distribution of decision factors in the two rice terraces was revealed as obviously different by statistical evidence $(\mathrm{P}<$ $0.05)$.

\subsection{Regional Characteristic of Decision Factors Presented by Non-Adjusted Odds Ratio by Topographic Conditions}

Figure 4 presents the result of sub-group analysis with the forest plot of the non-adjusted OR for each decision factor by topographic sub-groups. P value was calculated from the statistical test for the non-adjusted OR in logistic regression. These results revealed that in both of the sites, the advantageous factors for intensive farming, area and road, were less related to topography and had less influence on the outcome of cultivation abandonment in the overall topographic sub-groups. However, the behavior of disadvantageous factors for intensive farming, the number of adjacent fields and soil erosion, were strongly related to topography. For example, focusing on the effect of the number of adjacent fields, this factor has a slight negative contribution to abandoning cultivation $\left(\mathrm{OR}=8.69 \mathrm{E}-01^{*}\right)$ within overall samples in Site A, while in the sub-group of gentle slopes (slope $\left.<9.94\right)$, this factor has an obviously negative contribution (OR $=5.35 \mathrm{E}-02 * *)$.

On the other hand, the number of adjacent fields in site B had a slightly positive influence for samples overall $\left(\mathrm{OR}=1.14 \mathrm{E}+00^{* * *}\right)$. In terms of the effect of soil erosion in Site A, it showed a positive contribution towards abandoning cultivation for samples overall $\left(\mathrm{OR}=1.28 \mathrm{E}+08^{* * * *}\right)$. Comparing the effect of soil erosion by topographic sub-groups in site A revealed its significance, especially in areas of upper elevation (Elevation $\geq$ 1312.12: $\mathrm{OR}=3.90 \mathrm{E}+03^{*}$ ) or steep slope (Slope $\geq 9.94$ : $\mathrm{OR}=2.66 \mathrm{E}+07^{* * * *}$ ). In site $\mathrm{B}$, the effects of soil erosion were observed as a positive contribution for samples overall $\left(\mathrm{OR}=4.25 \mathrm{E}+04^{* * * *}\right)$, whereas for upper elevation sub-groups, it presented a negative contribution with statistical evidence (Elevation $\geq 1164.07$ : OR = $2.31 \mathrm{E}-01 *)$.

\subsection{Influence of Cultural Endemism on Decision Factors Presented by Adjusted Odds Ratio by Topographic Conditions}

The results of the Mann-Whitney $U$ test for baseline topographic conditions of regrouped samples divided by each factor's median value are shown in Figure 5. The topographic confounding variables for each decision factor were detected, presenting statistical evidence $(\mathrm{P}<0.05)$. Inspection of Figure 5 reveals the existence of some interaction between topographic conditions and whole decision factors. This evidence is consistent with the general consideration of prior research that addresses the linkage between topography and cultivation abandonment (Topography). However, dealing with each decision factor individually revealed different topographic confounders for each decision factor. That is, a significant interaction with elevation was detected for all decision factors in both sites. On the other hand, the significant interaction with slope was detected for every factor except for area and soil erosion in site B. These detected confounders were used to adjust the OR in interaction 
NON-ADAJUSTED ODDS RATIO OF EACH DECISION FACTOR BY TOPOGRAPHIC SUB-GROUPS



\section{Site B: Rain-fed rice terrace}

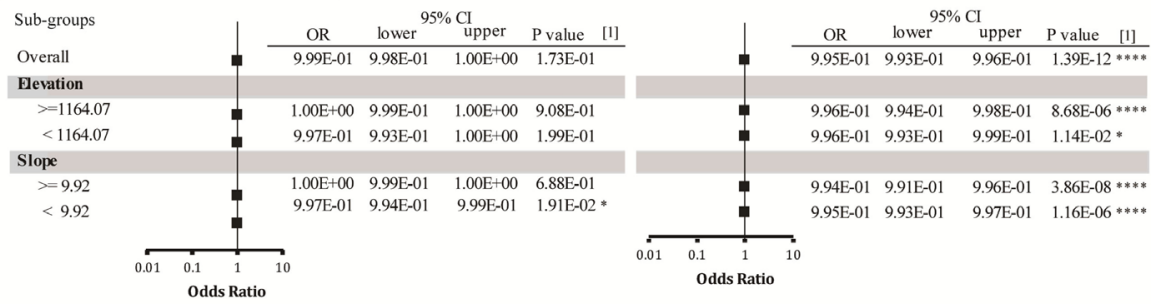

\section{Disavantageous factors}

3) Number of adjacent fields 4) Soil erosion

\section{Site A: Irrigated rice terrace}

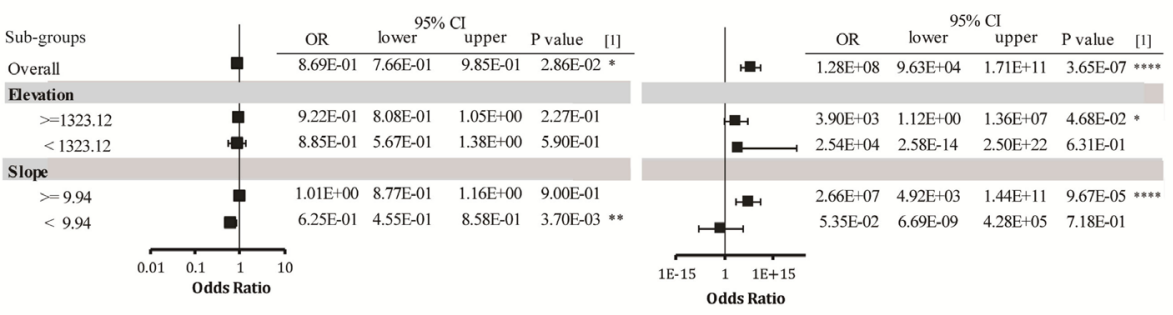

\section{Site B: Rain-fed rice terrace}
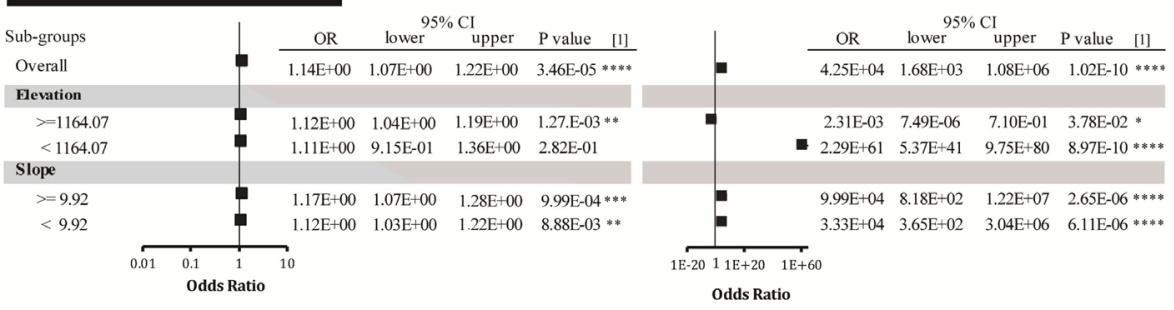

[1] Symbols shows the scale of $P$ value:

$\because ?$ means $\mathrm{P}<0.01$, '*' means $\mathrm{P}<0.05$, '**' means $\mathrm{P}<0.01$, '***' means $\mathrm{P}<0.001$, '****' means $\mathrm{P}<0.0001$

Figure 4. Non-adjusted odds ratio of each decision factor by topographic conditions.

analysis for each decision factor to remove the effect of topography. The OR of each decision factor was adjusted by the detected topographic confounders (see Figure 6). The resulting adjusted OR values were plotted on a forest plot, which presented the magnitude of decision factors' effect by their distance from 1 . As the Figure 6 shows, in Site A, there were no apparent effects of any decision factors. In site B, however, the results revealed the powerful effect of particular decision factors: the "distance to roads" factor's fairly negative contribution on abandoning cultivation ( $\left.\mathrm{OR}=9.94 \mathrm{E}-01^{* * * *}\right)$, as well as the "number of adjacent field" factor's positive contribution on abandoning cultivation $\left(\mathrm{OR}=1.13 \mathrm{E}+00^{* * *}\right)$. 
Elevation (meter) Slope (degree)

Subgroups

(Number of samples)

Site A: Irrigated rice terrace

Area

$>=457.68(360)$

$<457.68(359)$

Distance to roads

$>=149.76$ (360)

$<149.76$ (359)

$-1-$


Number of adjacent fields

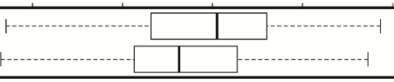

$>=4$

$<4$

(271)
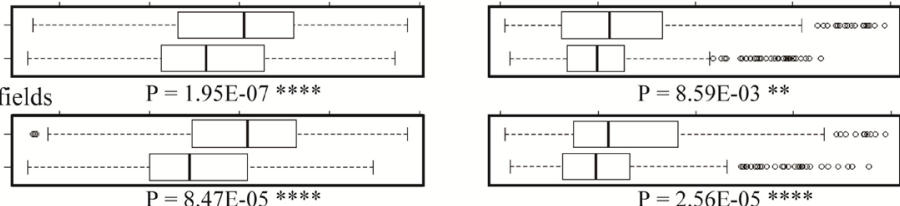

Soil erosion

$>=0.046 \quad(360)$

$<0.046$

(359)
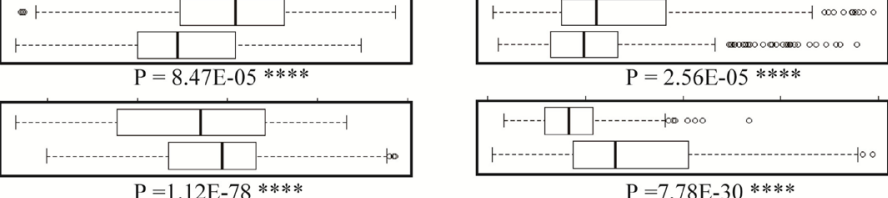

$\mathrm{P}=2.56 \mathrm{E}-05 * * * *$

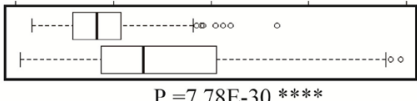

Subgroups

(Number of samples)

Area

Site B: Rain-fed rice terrace

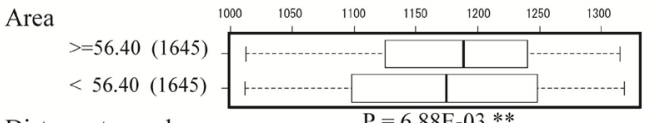

Distance to roads

$>=314.91$ (1645)

$<314.91(1645)$

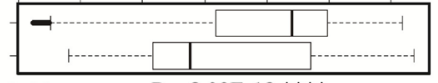

Number of adjacent fields

$>=4 \quad$ (992)

$<\quad$ (2298)
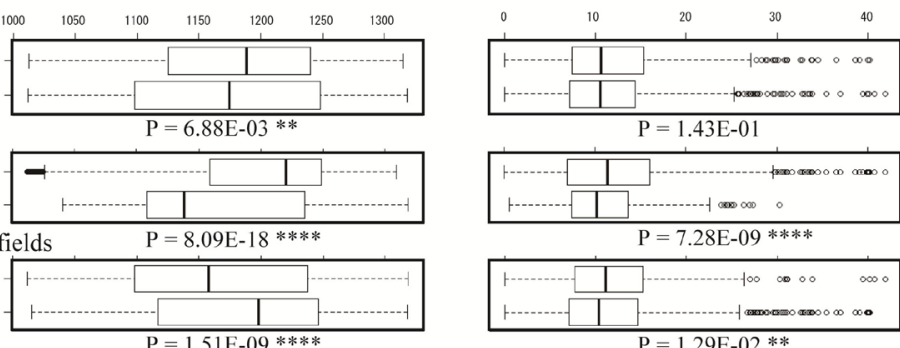

Soil erosion

$>=0.11 \quad(1645)$

$<0.11(1645)$
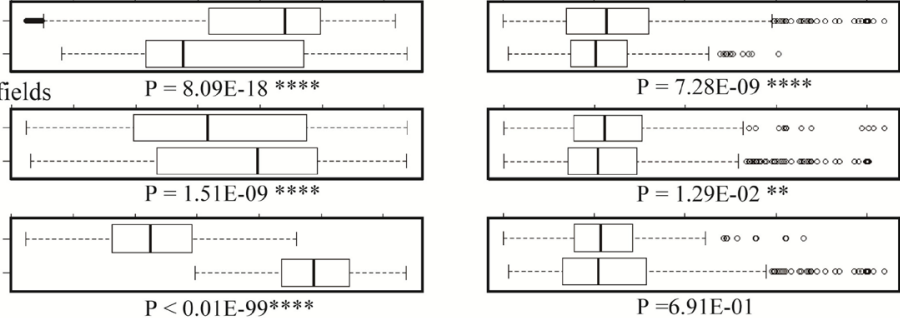

$\mathrm{P}=1.29 \mathrm{E}-02 * *$

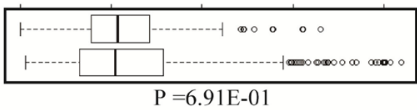

Symbols shows the scale of $\mathrm{P}$ value which was calculated from interaction:

' 'means $\mathrm{P}<0.01$, '*' means $\mathrm{P}<0.05$, '**' means $\mathrm{P}<0.01$, '***' means $\mathrm{P}<0.001$, '*****' means $\mathrm{P}<0.0001$

Figure 5. Baseline characteristics of topography for each decision factor.

\section{ADJUSTED ODDS RATIO OF EACH DECISION FACTOR}

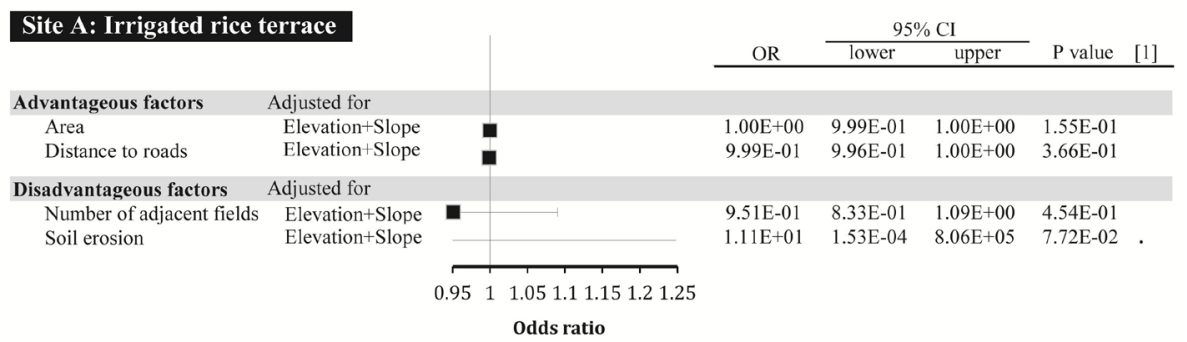

Site B: Rain-fed rice terrace

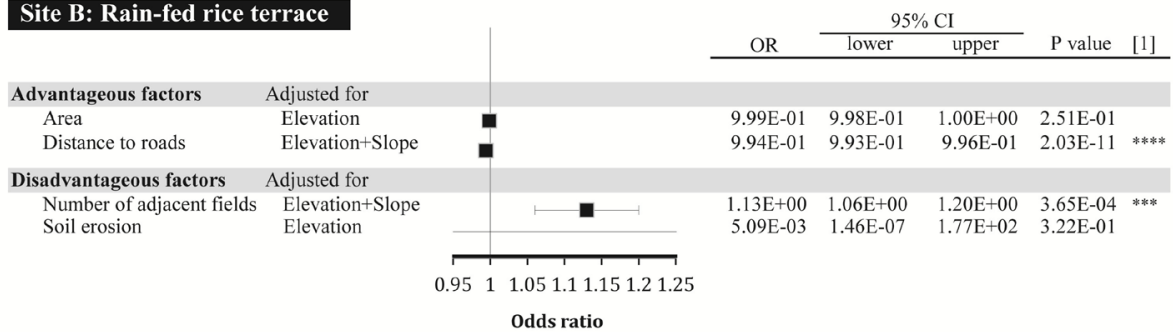

[1] Symbols shows the scale of $\mathbf{P}$ value:

'.' means $\mathrm{P}<0.01$, ‘*' means $\mathrm{P}<0.05$, '**' means $\mathrm{P}<0.01$, '***' means $\mathrm{P}<0.001$, '****' means $\mathrm{P}<0.0001$

Figure 6. Effect of the decision factors adjusted by topography. 


\section{Discussion}

\subsection{Effect of Toraja's Cultural Endemism on Decision-Making about Rice Cultivation}

Abandonment of cultivation in mountainous regions is an expanding trend worldwide, and an urgent practical policy recommendation to stop the spread of cultivation abandonment is needed. However, the discussion about the factors of the expanding trend of abandoned cultivation so far have placed a disproportionate weight on universal factors and lacked evaluation of the regionality of the phenomenon, which appears on a small scale as a reflection of culture, micro-topography, or ecosystem functions that benefit local people on the regional scale. However, because the situation of abandoning cultivation in mountainous regions differs regionally, it cannot be explained equally in different places by the above-mentioned factors.

Based on the assumptions that follows from the causal framework constructed by the primary observations, this paper quantitatively evaluated regional characteristics and the influence of cultural endemism on decision-making about abandoning cultivation. The OR was calculated for each decision factor, which were divided by the terms of interest, advantageous or disadvantageous factors for intensive farming. Two rice terraces with different water systems—Site A, an irrigated field, and site B, a rain-fed field—were examined to compare the results of analysis.

The results of the non-adjusted OR detected the regional characteristics of decision factors' effects by topographic condition (see Figure 4). Contrary to arguments from prior studies, the regional characteristics of decision-making indicated that advantageous factors for intensive farming related to labor intensity, area, and distance to roads, had no distinctive effect on decision-making in both sites. Moreover, the effects of disadvantageous factors on intensive farming — number of adjacent fields and soil erosion—were detected in both sites.

As evident in Figure 5, some topographic confounders for all decision factors emerged. This was consistent with arguments of prior research that referred to topographic conditions as the factor leading to abandoning cultivation [11]. However, this study's results also revealed an unequal effect by topographic confounders on each decision factor (see Figure 5). In order to determine the influence of cultural endemism on decision-making, the effects of topographic confounders for each decision factor were adjusted through interaction analysis of the logistic regression model. The results of adjusted OR showed that the effect of all decision factors in irrigated rice terraces were cancelled out (see Figure 6). It indicated that decision-making about cultivation abandonment of irrigated rice terraces was simply based on the topographic environment. On the other hand, the results from the rain-fed rice terrace showed apparent effects of cultural endemism in addition to those from topography. To be more precise, the results indicated that the greater the distance to the road, the less frequently cultivation abandonment occurred. Additionally, the results also indicated that the larger the number of adjacent fields, the more frequently cultivation abandonment occurred.

To interpret the detected influence of cultural endemism on decisions, however, comparison of the actual cultural differences between two rice terraces is required, which was revealed by the interviews and field observations (see Table 2). The fact that the results showed the effect of cultural endemism on the disadvantageous factors for intensive farming rather than the advantageous factors may be interpreted as a result of the regulation of customary laws that clarifies upper land owners' responsibility for the slope failure of rice terraces' footpaths and for soil erosion. Moreover, in Toraja society, there are two alternate inheritance systems: single inheritance, as seen in Site A, and divided succession, as seen in site B. The significant difference in rice field area between Site A and Site B was thought to be a result of the different inheritance systems. Therefore, in rain-fed rice terraces where the standard area of each fields is small, it may be reasonable to decide to abandon cultivation under the condition of a high number of adjacent fields, considering the costs versus benefits of farming. Moreover, the difference of harvested rice consumption also might be a factor in local people's negligence in terms of intensity, an advantageous factor for intensive farming in rain-fed rice terraces.

In this way, because the results had explanations from actual cultural endemism, it seems reasonable to conclude that this is a compelling method to detect the influence of cultural endemism on decision-making about abandoning cultivation in Toraja region.

While prior research about the Toraja region has focused on the relation of ritual and rice cultivation [5] or the centrality of rice cultivation on the Toraja people's livelihood [6], there has been no study that tried to explore the influence of culture and rituals on the local people's decision-making for cultivation. Therefore, this is the first study to provide initial quantitative evidence on the influence of cultural endemism on decision-making about cultivation abandonment. 
In addition, by taking cultural endemism, such as the customary law, communal-inherent systems and the ritual value of indigenous rice in rain-fed terraces, into consideration, it can present convincing evidence for behavior science's argument about collective action: the motivation to join collective acts is influenced by the perceived value placed on the outcome [7] [8]. Therefore, these results indicate the importance of considering cultural endemism by assessing regionality of causal factors on abandonment of cultivation to generate practical policy recommendations about cultivation abandonment.

\subsection{Application of an Observation-Oriented Approach to Discover the Causal Association}

The present study demonstrated the effects of cultural endemism on decisions about abandoning cultivation by integrating qualitative field observations and interviews and quantitative evaluation by estimating OR values using logistic regression. One of the attractive originalities of this research is its observation-oriented approach, which constructs its causal framework based on a primary on-site survey prior to statistical causal discoveries. This approach enabled catching hidden causality, which would be overlooked with data-intensive approaches.

In general, it is difficult to discuss analysis results of observational research because of spatial selection bias. However, this paper discovered the causal effects of unmeasured cultural endemism by controlling overt covariate through topographic data generation using GIS and radar remote sensing techniques. Though radar techniques are generally used to observe micro-environmental changes such as ground displacement on a broad scale, this research applied these techniques to analyze the factors associated with local people's decision-making in a regional scale. In this way, this research demonstrated the efficiency of geo-spatial data for controlling the confounders in an observational research based on an on-site survey.

However, it is important to recognize that the primary constructed framework includes the arbitrariness of the observer simultaneously; that is, it may exclude other hidden covariates that were not detected by observation. Consequently, it is pointless to conclude that the present results reveal the complete causal associations of decision-making about cultivation abandonment in the Toraja region. However, discovering the causal associations singly by adjusting for the quantified overt covariate detected by observation is an important process for scientifically clarifying the mechanism of regionality of cultivation abandonment. Future work should therefore include follow-up on-site investigations to determine the further underlying causal associations with regional decision-making for abandoning cultivation.

\section{Conclusion}

The regional characteristics of decision-making about cultivation abandonment were explained by evaluation of the odds ratio of particular decision factors that were detected by field observations and interviews. Contrary to prior research, the results revealed that decisions about abandoning cultivation in Toraja were greatly associated with disadvantageous factors for intensive farming, rather than advantageous factors. Moreover, the magnitude of disadvantageous factors' effects differs by topographic condition. Assuming these regional characteristics of decision-making was simultaneously influenced by topography and cultural endemism, the effects from topographic confounders were removed by estimating the adjusted odds ratio in order to detect the influence from cultural endemism alone. The results indicated that decision-making about cultivation abandonment in irrigated rice terraces was due to simple topographic conditions, whereas decision-making in rain-fed rice terrace was due to cultural endemism combined with topographic conditions. Given the evidence from the explanation of the results about cultural endemism for each site, therefore, it could be concluded that the detection of cultural endemism's influence was achieved using the algorithm which this paper proposed. These findings extend the discussion about the causality of abandoning cultivation to the regional scale, referring to the regional characteristics of decision-making as well as the influence cultural endemism exerts on such decision-making. However, future work is still required to conduct further on-site surveys in order to ascertain the underlying causal associations of regionalism on abandoning cultivation.

\section{Acknowledgements}

This work was supported by JSPS KAKENHI Grant Number 13J02903, JSPS International Training Program (ITP) and Murata Science Foundation. 


\section{References}

[1] Kozak, J., Ostapowicz, K., Szablowska-Midor, A. and Widacki, W. (2004) Land Abandonment in the Western Beskidy Mts and Its Environmental Background. Ekológia (Bratislava), 23, 116-126.

[2] Tatoni, T. and Roche, P. (1994) Comparison of Old-Field and Forest Revegetation Dynamics in Provence. Journal of Vegetation Science, 5, 295-302. http://dx.doi.org/10.2307/3235852

[3] Le Houérou, H.N. (1993) Land Degradation in Mediterranean Europe: Can Agroforestry Be a Part of the Solution? A Prospective Review. Agroforestry Systems, 21, 43-61. http://dx.doi.org/10.1007/bf00704925

[4] MacDonald, D., et al. (2000) Agricultural Abandonment in Mountain Areas of Europe: Environmental Consequences and Policy Response. Journal of Environmental Management, 59, 47-69. http://dx.doi.org/10.1006/jema.1999.0335

[5] Hosoda, A. (2006) Traditional and Institutional Characteristics in Rural Society of Tana Toraja. KEIZAI SHIRIN The Hosei Univ. Econ. Rev.73, 245-274.

[6] Oide, A., Doko, T. and Chen, W. (2015) Network Analysis of Livelihood Pattern Changes in North Toraja, Indonesia, 1975-2014. Papers on Environmental Information Science, 29, 339-344.

[7] Giguère, B. and Lalonde, R.N. (2010) Why Do Students Strike? Direct and Indirect Determinants of Collective Action Participation. Political Psychology, 31, 227-247. http://dx.doi.org/10.1111/j.1467-9221.2009.00750.x

[8] Louis, W.R., Taylor, D.M. and Neil, T. (2004) Cost-Benefit Analyses for Your Group and Yourself: the Rationality of Decision-Making in Conflict. International Journal of Conflict Management, 15, 110-143.

http://dx.doi.org/10.1108/eb022909

[9] Matsuno, Y., et al. (2006) Prospects for Multifunctionality of Paddy Rice Cultivation in Japan and Other Countries in Monsoon Asia. Paddy and Water Environment, 4, 189-197. http://dx.doi.org/10.1007/s10333-006-0048-4

[10] Molden, D., Rijsberman, F., Matsuno, Y. and Amarasinghe, U. (2001) Increasing Productivity of Water : A Requirement for Food and Environmental Security. 22-23.

[11] Mottet, A., Ladet, S., Coqué, N. and Gibon, A. (2006) Agricultural Land-Use Change and Its Drivers in Mountain Landscaps: A Case Study in the Pyrenees. Agriculture, Ecosystems \& Environment, 114, 296-310. http://dx.doi.org/10.1016/j.agee.2005.11.017

[12] Bakker, M.M., et al. (2005) Soil Erosion as a Driver of land-Use Change. Agriculture, Ecosystems \& Environment, 105, 467-481. http://dx.doi.org/10.1016/j.agee.2004.07.009

[13] Bürgi, M. and Turner, M.G. (2002) Factors and Processes Shaping Land Cover and Land Cover Changes along the Wisconsin River. Ecosystems, 5, 184-201. http://dx.doi.org/10.1007/s10021-001-0064-6

[14] Gisbert, J.M., Ibanez, S. and Perez, M.A. (2005) Terrace Abandonment in the Ceta Valley, Alicante Province, Spain. Adv Geoecol, 36, 329-337.

[15] Nagendra, H., Southworth, J. and Tucker, C. (2003) Accessibility as a Determinant of Landscape Transformation in Western Honduras: Linking Pattern and Process. Landscape Ecology, 18, 141-158. http://dx.doi.org/10.1023/A:1024430026953

[16] Hatna, E. and Bakker, M.M. (2011) Abandonment and Expansion of Arable Land in Europe. Ecosystems, 14, $720-731$. http://dx.doi.org/10.1007/s10021-011-9441-y

[17] Rosenbaum, P.R. and Rubin, D.B. (1984) Reducing Bias in Observational Studies Using Sub-Classification on the Propensity Score. Journal of the American Statistical Association, 79, 516-524. http://dx.doi.org/10.1080/01621459.1984.10478078

[18] Cochran, W.G. and Chambers, S.P. (1965) The Planning of Observational Studies of Human Populations. Journal of the Royal Statistical Society: Series A, 128, 234-255. http://dx.doi.org/10.2307/2344179

[19] Morgan, S.L. (2010) A Quantitative Tour of the Social Sciences. Contemporary Sociology: A Journal of Review, 39, 164-166.

[20] Wegmuller, U., Werner, C. and Strozzi, T. (1998) SAR Interferometric and Differential Interferometric Processing Chain. Proceedings of the 1998 IEEE International Geoscience and Remote Sensing Symposium, Seattle, 6-10 July 1998, 1106-1108. http://dx.doi.org/10.1109/IGARSS.1998.699687

[21] Lowry, B., et al. (2013) High Resolution Displacement Monitoring of a Slow Velocity Landslide Using Ground Based Radar Interferometry. Engineering Geology, 166, 160-169. http://dx.doi.org/10.1016/j.enggeo.2013.07.007

[22] Sousa, J.J., et al. (2010) PS-InSAR Processing Methodologies in the Detection of Field Surface Deformation-Study of the Granada Basin (Central Betic Cordilleras, Southern Spain). Journal of Geodynamics, 49, 181-189. http://dx.doi.org/10.1016/j.jog.2009.12.002

[23] Sun, Q., et al. (2015) Slope Deformation Prior to Zhouqu, China Landslide from InSAR Time Series Analysis. Remote Sensing of Environment, 156, 45-57. http://dx.doi.org/10.1016/j.rse.2014.09.029 
[24] Liu, L., Zhang, T. and Wahr, J. (2010) InSAR Measurements of Surface Deformation over Permafrost on the North Slope of Alaska. Journal of Geophysical Research, 115, F03023. http://dx.doi.org/10.1029/2009jf001547

[25] Cox, D.R. (1958) The Regression Analysis of Binary Sequences. Journal of the Royal Statistical Society: Series B, 20, 215-242.

[26] Szklo, M. (Moyses) and Nieto, F.J. (2007) Epidemiology: Beyond the Basics. Jones and Bartlett Publishers, Inc., Sudbury. 